\title{
Minimax Adjustment of a Univariate Distribution to Satisfy Componentwise Bounds and/or Ranking
}

\author{
A. J. Goldman \\ Institute for Basic Standards, National Bureau of Standards, Washington, D.C. 20234
}

(May 7, 1969)

\begin{abstract}
Consider a discrete probability distribution, represented by an $n$-vector a. This paper treats the problem of adjusting a as little as possible, in the sense of minimizing $\max _{i}\left|x_{i}-a_{i}\right|$, to obtain a distribution $\mathbf{x}$ which satisfies given component wise bounds $\mathbf{L} \leqslant \mathbf{x} \leqslant \mathbf{U}$, or a given component wise ranking, or both. The resulting linear programs are shown to admit special explicit solution algorithms.
\end{abstract}

Key words: Linear programs; mathematical models; minimax estimation; operations research; probability distribution.

\section{Introduction}

An $n$-vector $\mathbf{x}$ will be called a probability vector if its components $x_{i}$ are nonnegative and sum to unity. This paper deals first with the following problem: given a probability $n$-vector a, and $n$-vectors $\mathbf{L}$ and $\mathbf{U}$, to find a probability $n$-vector $\mathbf{x}$ which minimizes

$$
F(\mathbf{x})=\max _{i}\left|x_{i}-a_{i}\right|
$$

subject to the constraints

$$
\mathbf{O} \leqslant \mathbf{L} \leqslant \mathbf{x} \leqslant \mathbf{U}
$$

For the problem to be feasible, it is obviously necessary that

$$
\begin{gathered}
L_{i} \leqslant U_{i} \quad(\text { all } i), \\
\Sigma_{i} L_{i} \leqslant 1 \leqslant \Sigma_{i} U_{i},
\end{gathered}
$$

and so these conditions are imposed at the outset.

The motivating situation is one in which values must be attributed to the components $x_{i}$ of an unknown discrete probability distribution. One type of information, e.g., data on some previous analogous situation, suggests the estimate a. Another, e.g., theoretical analyses or subjective opinions on the "present" situation, imposes the componentwise bounds (1.2). If a does not satisfy (1.2), a common procedure is to "adjust a as little as possible" so as to satisfy (1.2). Here the minimization of $F(\mathbf{x})$ is taken to express the "as little as possible" criterion in replacing a by a suitable $\mathbf{x}$.

After some preliminaries are disposed of in section 2, a solution method for this problem is presented in section 3. In fact, the method is developed for the more general version in which (1.1) is replaced by

$$
F(\mathbf{x})=\max _{i}\left\{w_{i}\left|x_{i}=a_{i}\right|\right\}
$$

where the $w_{i}$ are prescribed positive "weights." This corresponds to the case in which the accuracy, with which $\mathbf{x}$ approximates $\mathbf{a}$, is more important for some components than for others. 
Section 4 considers the analogous problem in which the componentwise-bound constraints (1.2) are replaced by a given componentwise ranking

$$
x_{1} \leqslant x_{2} \leqslant \ldots \leqslant x_{n} .
$$

Then, in section 5, the problem with both bounds and ranking is treated. (Both of these sections deal only with the "unweighted" objective function (1.1); the weighted version can be handled as a linear program, but our concern is with more explicit methods.)

Related work is found in $[1,2,3]^{1}$. The present paper, though self-contained, isolates in a form more convenient for reference some material appearing in [1].

\section{Preliminaries}

This section contains solution methods for four subproblems which will arise later. The material is presented for the sake of completeness; the same or similar problems have surely arisen in the literature as phases in other optimization analyses. In each case below, the solution method provides a constructive proof that certain obviously necessary conditions, for the existence of solutions, are also sufficient.

Problem I. Given $n$-vectors $\mathbf{A}$ and $\mathbf{B}$, and number $S$, find an $n$-vector $\mathbf{y}$ such that

$$
\begin{gathered}
\mathbf{A} \leqslant \mathbf{y} \leqslant \mathbf{B}, \\
\Sigma_{i} y_{i}=S .
\end{gathered}
$$

The conditions

$$
\begin{gathered}
\mathbf{A} \leqslant \mathbf{B}, \\
\Sigma_{i} A_{i} \leqslant S \leqslant \Sigma_{i} B_{i},
\end{gathered}
$$

which are clearly necessary for Problem I to have a solution, will be assumed to hold. If $S=\Sigma_{i} B_{i}$ then $\mathbf{y}=\mathbf{B}$ is clearly a solution, so we assume $S<\Sigma_{i} B_{i}$. Then $k=n$ has the property

$$
\Sigma_{j \leqslant k} B_{j}+\Sigma_{j>k} A_{j}>S,
$$

but $k=0$ does not, and so there is a smallest $k \epsilon\{1,2, \ldots, n\}$ with this property. For that $k$, not only (2.5) but also

holds. Now set

$$
\Sigma_{j<k} B_{j}+\Sigma_{j \geqslant k} A_{j} \leqslant S
$$

$$
\begin{array}{ll}
y_{j}=B_{j} & \text { for } j<k, \\
y_{j}=A_{j} & \text { for } j>k, \\
y_{k}=S-\dot{\Sigma}_{j \neq k} y_{j} ; &
\end{array}
$$

that $A_{k} \leqslant y_{k} \leqslant B_{k}$ follows from (2.5) and (2.6).

Problem II. Given $n$-vector $\mathbf{Z}$, positive $n$-vector $\mathbf{w}$, and number $S$, find the minimum value $z^{*}$ of $z$ such that $z \geqslant 0$ and

$$
\sum_{1}^{n} \max \left\{0, Z_{i}-z / w_{i}\right\} \leqslant S .
$$

The condition $S \geqslant 0$, which is clearly necessary for Problem II to have a solution, will be assumed. Moreover, if $S=0$, then each of the nonnegative summands in (2.7) would have to vanish, yielding

$$
z^{*}=\max \left\{0, \max _{i} w_{i} Z_{i}\right\}
$$

as the solution; thus from now on we assume $S>0$. Furthermore if $Z_{i} \leqslant 0$, then the $i$ th summand in (2.7) will vanish for every $z \geqslant 0$, and so such $Z_{i}$ can be deleted from the problem in advance; if none are left then clearly $z^{*}=0$ is the solution. So $Z_{i}>0$ will be assumed.

${ }^{1}$ Figures in brackets indicate the literature references at the end of the paper. 
Now choose $Z_{n+1}=0$ and any $w_{n+1}>0$, renumber so that

$$
w_{1} Z_{1} \geqslant w_{2} Z_{2} \geqslant \cdots \geqslant w_{n} Z_{n}>w_{n+1} Z_{n+1}=0,
$$

and set

$$
Z_{j}^{*}=\Sigma_{i}^{i} Z_{i}-w_{j} Z_{j} \Sigma_{j}\left(1 / w_{i}\right)
$$

The sequence $\left\{Z_{j}^{*}\right\}_{1}^{n+1}$ is given by the recursion

$$
Z_{j+1}^{*}=Z_{j}^{*}+\left(w_{j} Z_{j}-w_{j+1} Z_{j+1}\right) \Sigma_{1}^{j}\left(1 / w_{i}\right)
$$

which shows it to be nondecreasing. And unless $\sum_{1}^{n} Z_{i}<S$ (in which case $z^{*}=0$ is the solution), we have

$$
Z_{1}^{*}=0<S \leqslant \Sigma_{1}^{n} Z_{i}=Z_{n+1}^{*}
$$

Thus there is a unique $J \epsilon\{1,2, \ldots, n\}$ such that

$$
\begin{aligned}
& 0=Z_{1}^{*} \leqslant Z_{2}^{*} \leqslant \ldots \leqslant Z_{J}^{*}<S \leqslant Z_{J+1}^{*} \leqslant \ldots \leqslant Z_{n+1}^{*} . \\
& \text { If } 0 \leqslant z<w_{J+1} Z_{J+1} \text {, then } \\
& \sum_{i} \max \left\{0, Z_{i}-z / w_{i}\right\} \geqslant \Sigma_{1}^{J+1} Z_{j}-z \sum_{1}^{J+1}\left(1 / w_{j}\right)>Z_{J+1}^{*} \geqslant S,
\end{aligned}
$$

so that $z$ does not satisfy (2.7). But if $w_{J+1} Z_{J+1} \leqslant z \leqslant w_{J} Z_{J}$, then (2.7) becomes

$$
\sum_{1}^{\prime} Z_{i}-z \sum_{1}^{\prime}\left(1 / w_{i}\right) \leqslant S
$$

which is equivalent to

$$
z \geqslant z^{*}=\left(\Sigma_{1} Z_{j}-S\right) / \Sigma_{1}^{J}\left(1 / w_{i}\right)
$$

By use of (2.8), the value of $z^{*}$ proposed in (2.9) is easily verified to satisfy $w_{J+1} Z_{J+1} \leqslant z \leqslant w_{J} Z_{J}$, and so is indeed the smallest $z \geqslant 0$ obeying (2.7).

Problem III. Given $n$-vector $\mathbf{Z}$, positive $n$-vector $\mathbf{w}$ and number $S$, find the minimum value $z^{* *}$ of $z$ such that $z \geqslant 0$ and

$$
\sum_{1}^{n} \min \left\{Z_{i}, z / w_{i}\right\} \geqslant S
$$

The condition

$$
\sum_{1}^{n} Z_{i} \geqslant S
$$

which is obviously necessary if (2.10) is to have a solution, will be assumed. If equality holds in (2.11), then for each $i$ the $i$ th summand in (2.10) must equal $Z_{i}$, so that the solution is

$$
z^{* *}=\max \left\{0, \max _{i} w_{i} Z_{i}\right\} ;
$$

thus from now on we assume strict inequality in (2.11). Moreover, if $Z_{i}<0$ then for any $z \geqslant 0$, $Z_{i}$ could be replaced by 0 on the left-hand side of $(2.10)$ without change in value; hence it can be assumed that all $Z_{i} \geqslant 0$. Now $z^{* *}=0$ will give the solution if $S \leqslant 0$, so we also assume $S>0$.

Choose $Z_{n+1}=0$ and any $w_{n+1}>0$, renumber so that

$$
w_{1} Z_{1} \geqslant w_{2} Z_{2} \geqslant \ldots . w_{n} Z_{n} \geqslant w_{n+1} Z_{n+1}=0,
$$

and set

$$
Z_{j}^{* *}=w_{j} Z_{j} \sum \dot{i}\left(1 / w_{i}\right)+\sum_{j+1}^{n} Z_{i}
$$


The sequence $\left\{Z_{j}^{* *}\right\}_{1}^{n+1}$ obeys the recursion

$$
Z_{j+1}^{* *}=Z_{j}^{* *}+\left(w_{j+1} Z_{j+1}-w_{j} Z_{j}\right) \Sigma_{i}\left(1 / w_{i}\right)
$$

and so is nonincreasing. Since

$$
Z_{n+1}^{* *}=0<S<\Sigma_{1}^{n} Z_{i}=Z_{1}^{* *},
$$

there is a unique $J \epsilon\{1,2, \ldots ., n\}$ such that

$$
Z_{1}^{*} \geqslant Z_{2}^{*} \geqslant . . \geqslant Z_{J}^{*} \geqslant S>Z_{J+1}^{*} \geqslant . . \geqslant Z_{n+1}^{*}=0 .
$$

Now if $z \leqslant w_{J+1}$, then

$$
\Sigma_{i} \min \left\{Z_{i}, z / w_{i}\right\} \leqslant z \Sigma_{1}^{J+1}\left(1 / w_{i}\right)+\Sigma_{J+2}^{n} Z_{1} \leqslant Z_{J+1}^{* *}<S
$$

so that $z$ does not satisfy (2.10). But if $w_{J+1} Z_{J+1} \leqslant z \leqslant w_{J} Z_{J}$, then (2.10) becomes

$$
z \Sigma_{1}^{J}\left(1 / w_{i}\right)+\sum_{J+1}^{n} Z_{j} \leqslant S
$$

which is equivalent to

$$
z \geqslant z^{* *}=\left(S-\Sigma_{J+1}^{n} Z_{j}\right) / \Sigma_{1}^{\prime}\left(1 / w_{i}\right) .
$$

By use of (2.12), the value of $z^{* *}$ proposed in (2.13) is easily verified to satisfy $w_{J+1} Z_{J+1} \leqslant z \leqslant w_{J} Z_{J}$, and so is indeed the smallest $z \geqslant 0$ obeying (2.10).

Problem IV. Given $n$-vectors $\mathbf{A}$ and $\mathbf{B}$, and number $S$, find an $n$-vector $\mathbf{y}$ such that

$$
\begin{gathered}
\mathbf{A} \leqslant \mathbf{y} \leqslant \mathbf{B}, \\
\sum_{i} y_{i}=S, \\
y_{1} \leqslant y_{2} \leqslant . . \leqslant y_{n} .
\end{gathered}
$$

Here it is convenient to define nondecreasing sequences $\left\{A_{i}^{\prime}\right\}_{1}^{n}$ and $\left\{B_{i}^{\prime}\right\}_{1}^{n}$, forming the components of respective vectors $\mathbf{A}^{\prime}$ and $\mathbf{B}^{\prime}$, by

$$
A_{i}^{\prime}=\max _{j \leqslant i} A_{j}, \quad B_{i}^{\prime}=\min _{j \geqslant i} B_{j} .
$$

Then (2.16) and (2.14) are readily proved equivalent to (2.16) and

$$
\mathbf{A}^{\prime} \leqslant \mathbf{y} \leqslant \mathbf{B}^{\prime}
$$

Thus necessary conditions, for Problem IV to have a solution, are

$$
\begin{gathered}
\mathbf{A}^{\prime} \leqslant \mathbf{B}^{\prime} \quad\left(\text { i.e. }, A_{i} \leqslant B_{j} \text { for } i \leqslant j\right), \\
\Sigma_{i} A_{i}^{\prime} \leqslant S \leqslant \Sigma_{i} B_{i}^{\prime} .
\end{gathered}
$$

These will be assumed to hold.

If $\mathbf{A}^{\prime}=\mathbf{B}^{\prime}$, then $\mathbf{y}=\mathbf{A}^{\prime}=\mathbf{B}^{\prime}$ is the solution. For $\mathbf{A}^{\prime} \neq \mathbf{B}^{\prime}$, define

$$
\theta=\left[S-\Sigma_{i} A_{i}^{\prime}\right] /\left[\Sigma_{i} B_{i}^{\prime}-\Sigma_{i} A_{i}^{\prime}\right]
$$

and set

$$
\mathbf{y}=\mathbf{A}^{\prime}+\theta\left(\mathbf{B}^{\prime}-\mathbf{A}^{\prime}\right) \text {. }
$$


Then (2.15) follows from the choice of $\theta$, and (2.14') becomes $0 \leqslant \theta \leqslant 1$, which follows from (2.19). As for (2.16),$i \leqslant j$ implies that $A_{i}^{\prime} \leqslant A_{j}^{\prime}$ and $B_{i}^{\prime} \leqslant B_{j}^{\prime}$, so that

$$
y_{i}=(1-\theta) A_{i}^{\prime}+\theta B_{i}^{\prime} \leqslant(1-\theta) A_{j}^{\prime}+\theta B_{j}^{\prime}=y_{j} .
$$

(The same approach yields a simpler solution method for Problem I than the one given above.)

\section{Solution for Componentwise Bounds}

We now return to the problem posed at the beginning of the paper, with objective function (1.5). It can be rephrased as the following linear program: choose a number $z$ and a probability $n$-vector $\mathbf{x}$, to minimize $z$ subject to the conditions

$$
\begin{gathered}
\mathbf{0} \leqslant \mathbf{L} \leqslant \mathbf{x} \leqslant \mathbf{U}, \\
z \geqslant w_{i}\left(x_{i}-a_{i}\right) \quad(\text { all } i), \\
z \geqslant w_{i}\left(a_{i}-x_{i}\right) \quad(\text { all } i) .
\end{gathered}
$$

The constraints of the linear program, including the requirement that $\mathbf{x}$ be a probability vector, can be written as follows:

$$
\begin{gathered}
\left.\max \left\{L_{i}, a_{i}-z / w_{i}\right\} \leqslant x_{i} \leqslant \min \left\{U_{i}, a_{i}+z / w_{i}\right\} \quad \text { (all } i\right) \\
\Sigma_{i} x_{i}=1
\end{gathered}
$$

A redundant constraint $z \geqslant 0$ can also be imposed. Thus the aim is to determine the smallest $z \geqslant 0$ for which the system (3.3a), (3.3b) has a solution $\mathbf{x}$.

For any fixed $z$, the system is an instance of Problem I in Section 2, with $S=1$ and

$$
A_{i}=\max \left\{L_{i}, a_{i}-z / w_{i}\right\}, \quad B_{i}=\min \left\{U_{i}, a_{i}+z / w_{i}\right\} .
$$

By the analysis in Section 2, a solution $\mathbf{x}$ exists if and only if

$$
\begin{gathered}
\max \left\{L_{i}, a_{i}-z / w_{i}\right\} \leqslant \min \left\{U_{i}, a_{i}+z / w_{i}\right\} \quad(\text { all } i), \\
\Sigma_{i} \max \left\{L_{i}, a_{i}-z / w_{i}\right\} \leqslant 1, \\
\Sigma_{i} \min \left\{U_{i}, a_{i}+z / w_{i}\right\} \geqslant 1 .
\end{gathered}
$$

So the objective is to determine the smallest value $z_{\min }$ of $z$ which will satisfy (3.4), (3.5) and (3.6).

Now the left-hand side in (3.4) is nonincreasing in $z$, while the right-hand side is nondecreasing. The left-hand sides of (3.5) and (3.6) are respectively nonincreasing and nondecreasing in $z$. It follows that, if

$$
\begin{aligned}
z^{\circ} & =\text { least } z \text { obeying }(3.4) \\
z^{*} & =\text { least nonnegative } z \text { obeying (3.5), } \\
z^{* *} & =\text { least nonnegative } z \text { obeying (3.6), }
\end{aligned}
$$

then

$$
z_{\min }=\max \left\{z^{\circ}, z^{*}, z^{* *}\right\}
$$

Since $L_{i} \leqslant U_{i},(3.4)$ reduces to

$$
\left.a_{i}-z / w_{i} \leqslant U_{i,} L_{i} \leqslant a_{i}+z / w_{i} \quad \text { (all } i\right),
$$

and so $z^{\circ}$ is readily determined as

$$
z^{\circ}=\max \left\{\max _{i} w_{i}\left(a_{i}-U_{i}\right), \max _{i} w_{i}\left(L_{i}-a_{i}\right)\right\} .
$$


Next, (3.5) can be rewritten

$$
\Sigma_{i} \max \left\{0,\left(a_{i}-L_{i}\right)-z / w_{i}\right\} \leqslant 1-\Sigma_{i} L_{i},
$$

so that determining $z^{*}$ is an instance of Problem II in Section 2, with

$$
\mathbf{Z}=\mathbf{a}-\mathbf{L}, \quad S=1-\Sigma_{i} L_{i} .
$$

The feasibility condition $S \geqslant 0$ is satisfied by virtue of the first part of (1.4).

Finally, (3.6) is equivalent to

$$
\Sigma_{i} \min \left\{U_{i}-a_{i}, z / w_{i}\right\} \geqslant 0,
$$

so that the determination of $z^{* *}$ is an instance of Problem III in Section 2, with

$$
\mathbf{Z}=\mathbf{U}-\mathbf{a}, \quad S=0 .
$$

The feasibility condition $\sum_{1}^{n} Z_{i} \geqslant S$ is satisfied by virtue of the second part of (1.4).

With $z^{\circ}, z^{*}$, and $z^{* *}$ determined, $z_{\min }$ can be found from (3.7). Then a single optimizing $\mathbf{x}$ can be found by applying, to the previously-mentioned instance of Problem I with $z=z_{\min }$, the solution method given in Section 2. Concerning the nonuniqueness of $\mathbf{x}$, compare Section 5 of [1].

\section{Solution for Componentwise Ranking}

The next problem to be considered is the determination of a probability $n$-vector $\mathbf{x}$, among those which obey the componentwise ranking

$$
x_{1} \leqslant x_{2} \leqslant \ldots . \leqslant x_{n},
$$

which minimizes

$$
F(\mathbf{x})=\max _{i}\left|x_{i}-a_{i}\right|
$$

This too can be reformulated as a linear program, namely to select a number $z$ and a vector $\mathbf{x} \geqslant 0$, so as to minimize $z$ subject to

$$
\begin{gathered}
\quad \sum_{i} x_{i}=1, \\
z \geqslant x_{i}-a_{i} \quad(\text { all } i), \\
z \geqslant a_{i}-x_{i} \quad(\text { all } i) .
\end{gathered}
$$

Conditions (4.3) and (4.4), together with $\mathbf{x} \geqslant 0$, can be abbreviated to

$$
\max \left\{0, a_{i}-z\right\} \leqslant x_{i} \leqslant a_{i}+z .
$$

A redundant constraint $z \geqslant 0$ can also be imposed. Thus the aim is to determine the smallest $z \geqslant 0$ for which the system (4.1), (4.2), (4.5) has a solution $\mathbf{x}$.

For any fixed $z \geqslant 0$, the system is an instance of Problem IV in Section 2, with $S=1$ and

$$
A_{i}=\max \left\{0, a_{i}-z\right\}, B_{i}=a_{i}+z .
$$

It is convenient to define vectors $\mathbf{a}^{*}$ and $\mathbf{a}^{* *}$, with nondecreasing component sequences given by

$$
a_{i}^{*}=\max _{j \leqslant i} a_{i}, a_{i}^{* *}=\min _{j \geqslant i} a_{i} .
$$

Then the vectors $\mathbf{A}^{\prime}$ and $\mathbf{B}^{\prime}$, described in Section 2's analysis of Problem 4, are given by

$$
A_{i}^{\prime}=\max \left\{0, a_{i}^{*}-z\right\}, B_{i}^{\prime}=a_{i}^{* *}+z .
$$


The conditions (2.18) and (2.19), for Problem IV to be feasible, become

$$
\begin{gathered}
\max \left\{0, a_{i}-z\right\} \leqslant a_{j}+z \text { for } i \leqslant j, \\
\Sigma_{i} \max \left\{0, a_{i}^{*}-z\right\} \leqslant 1 \leqslant \Sigma_{i}\left(a_{i}^{* *}+z\right) .
\end{gathered}
$$

Now the objective is to find $z_{\min }$, the smallest $z \geqslant 0$ satisfying (4.6) and (4.7). Arguing as in Section 3 , one finds that if

$$
\begin{aligned}
z^{0} & =\text { least nonnegative } z \text { obeying (4.6), } \\
z^{*} & =\text { least nonnegative } z \text { obeying first part of (4.7), } \\
z^{* *} & =\text { least nonnegative } z \text { obeying second part of (4.7), }
\end{aligned}
$$

then

$$
z_{\min }=\max \left\{z^{0}, z^{*}, z^{* *}\right\}
$$

Since $z \geqslant 0$ and each $a_{j} \geqslant 0, z^{0}$ is readily determined from (4.6) as

$$
z^{0}=\max \left\{0, \max _{i \leq j}\left(a_{i}-a_{j}\right) / 2\right\} .
$$

Since $a_{i}^{* *} \leqslant a_{i}$, implying

$$
\sum_{i} a_{i}^{* *} \leqslant \Sigma_{i} a_{i}=1
$$

$z^{* *}$ is readily determined from $(4.7)$ as

$$
z^{* *}=\left(1-\Sigma_{i} a_{i}^{* *}\right) / n .
$$

Finally, the determination of $z^{*}$ is an instance of Problem II, with $S=1, w_{i}=1$, and $Z_{i}=a_{i}^{*}$.

\section{Solution for Componentwise Bounds and Ranking}

The final version to be treated is the determination of a probability $n$-vector $\mathbf{x}$ which minimizes $\max _{i}\left|x_{i}-a_{i}\right|$, subject both to the ranking condition

$$
x_{1} \leqslant x_{2} \leqslant \ldots \leqslant x_{n},
$$

and to the componentwise bounds

$$
\mathbf{O} \leqslant \mathbf{L} \leqslant \mathbf{x} \leqslant \mathbf{U} .
$$

The analysis proceeds much as in the preceding Section, with (4.5) replaced by

$$
\max \left\{L_{i}, a_{i}-z\right\} \leqslant x_{i} \leqslant \min \left\{U_{i}, a_{i}+z\right\} .
$$

Again we have an instance of Problem IV, with $S=1$, and with

$$
A_{i}=\max \left\{L_{i}, a_{i}-z\right\}, \quad B_{i}=\min \left\{U_{i}, a_{i}+z\right\} .
$$

As before, set

$$
a_{i}^{*}=\max _{j \leqslant i} a_{j}, a_{i}^{* *}=\min _{j \geqslant i} a_{j},
$$

and also put

$$
L_{i}^{\prime}=\max _{j \leqslant i} L_{j}, \quad U_{i}^{\prime}=\min _{j \geqslant i} U_{j} .
$$


Then the vectors $\mathbf{A}^{\prime}$ and $\mathbf{B}^{\prime}$ are now given by

$$
A_{i}^{\prime}=\max \left\{L_{i}^{\prime}, a_{i}^{*}-z\right\}, \quad B_{i}^{\prime}=\min \left\{U_{i}^{\prime}, a_{i}^{* *}+z\right\} .
$$

We continue to have

$$
z_{\min }=\max \left\{z^{0}, z^{*}, z^{* *}\right\}
$$

where

$$
\begin{aligned}
z^{0} & =\text { least } z \text { for which } \mathbf{A}^{\prime} \leqslant \mathbf{B}^{\prime}, \\
z^{*} & =\text { least nonnegative } z \text { for which } \Sigma_{i} A_{i}^{\prime} \leqslant 1, \\
z^{* *} & =\text { least nonnegative } z \text { for which } \Sigma_{i} B_{i}^{\prime} \geqslant 1 .
\end{aligned}
$$

The problem defining $z^{0}$, and hence the overall problem, has a solution only if

$$
L_{i} \leqslant U_{j} \quad \text { for } i \leqslant j .
$$

Assuming this holds, the remaining conditions defining $z^{0}$ are (for $i \leqslant j$ )

and so

$$
L_{i} \leqslant a_{j}+z, a_{i}-z \leqslant U_{j}, a_{i}-z \leqslant a_{j}+z,
$$

$$
z^{0}=\max _{i \leqslant j} \max \left\{L_{i}-a_{j}, a_{i}-U_{j},\left(a_{i}-a_{j}\right) / 2\right\} .
$$

The condition defining $z^{*}$ reads

$$
\Sigma_{i} \max \left\{L_{i}^{\prime}, a_{i}^{*}-z\right\} \leqslant 1,
$$

which can be rewritten

$$
\Sigma_{i} \max \left\{0,\left(a_{i}^{*}-L_{i}^{\prime}\right)-z\right\} \leqslant 1-\Sigma_{i} L_{i}^{\prime} .
$$

This is an instance of Problem II, with $w_{i}=1$,

$$
Z_{i}=a_{i}^{*}-L_{i}^{\prime}, \quad S=1-\Sigma_{i} L_{i}^{\prime} .
$$

The consistency condition $S \geqslant 0$, i.e.,

$$
\Sigma_{i} L_{i}^{\prime} \leqslant 1
$$

is required for a solution to exist.

The condition defining $z^{* *}$ reads

$$
\Sigma_{i} \min \left\{U_{i}^{\prime}, a_{i}^{* *}+z\right\} \geqslant 1,
$$

which can be rewritten

$$
\Sigma_{i} \min \left\{U_{i}^{\prime}-a_{i}^{* *}, z\right\} \geqslant 1-\Sigma_{i} a_{i}^{* *} .
$$

This is an instance of Problem III, with $w_{i}=1$,

$$
Z_{i}=U_{i}^{\prime}-a_{i}^{* *}, \quad S=1-\Sigma_{i} a_{i}^{* *} .
$$

The consistency condition $\Sigma_{i} Z_{i} \geqslant S$, i.e.,

$$
\Sigma_{i} U_{i}^{\prime} \geqslant 1
$$

is required for a solution to exist.

Thus the conditions on the data $\mathbf{L}$ and $\mathbf{U}$, for the componentwise bounds and ranking to be consistent, are given by (5.4), (5.6) and (5.7). 


\section{References}

[1] Goldman, A. J., and Meyers, P. R., Minimax error selection of a discrete univariate distribution with prescribed componentwise bounds, J. Res. NBS 72B (Math. Sci.), No. 4, 263-271 (1968).

[2] Goldman, A. J., Minimax error selection of a discrete univariate distribution with prescribed componentwise ranking, J. Res. NBS 72B (Math. Sci.), No. 4, 273-277 (1968).

[3] Goldman, A. J., Minimax error selection of a univariate distribution with prescribed componentwise bounds and ranking, J. Res. Nat. Bur. Stand. (U.S.), 73B (3), 225-230 (1969).

(Paper 73B3-303) 